\title{
An Analytical Model of the Dynamics of the Liquefied Vitreous Induced by Saccadic Eye Movements
}

\author{
RODOLFO REPETTO* \\ Dipartimento di Ingegneria delle Strutture, delle Acque e del Terreno, University of L'Aquila, \\ Monteluco di Roio, 67040, L'Aquila, Italy
}

(Received: 11 December 2004; accepted in revised form: 6 June 2005)

\begin{abstract}
An analytical model of the dynamics of the vitreous humour induced by saccadic movements within the eye globe is presented. The vitreous is treated as a weakly viscous Newtonian incompressible fluid, an assumption which is appropriate when the vitreous is liquefied or when it is replaced by aqueous humour after surgery. The thin viscous boundary layer generated during a saccadic movement on the side wall is neglected and the flow field is assumed to be irrotational. The vitreous chamber is described as a weakly deformed sphere and this assumption allows a linear treatment of the problem. An analytical solution is found in the form of an expansion of spherical harmonics. Results show that the non-spherical shape of the container generates a flow field characterised by significant velocities and strong three-dimensionality. The model allows the computation of the dynamic pressure on the wall, which may play a role in the generation of retinal detachments. Moreover, results suggest that the irregular shape of the globe may significantly modify tangential stresses on the boundary with respect to the case of motion within a sphere. A simplified analytical solution, for the case of two-dimensional flow within an impulsively rotated container, shows that boundary layer detachment is expected to occur for angles of rotation larger than a threshold value of $15^{\circ}$ circa.
\end{abstract}

Key words: Eye, Vitreous dynamics, Biological fluid dynamics, Irrotational flow, Fluid mechanics.

\section{Introduction}

The human eye consists of two main chambers (see Figure 1): the anterior chamber, enclosed by the cornea and the lens, and the vitreous chamber, which is the space bounded by the lens and the retina. The anterior chamber contains the aqueous humour, a physiological liquid whose mechanical characteristics are similar to those of water; the vitreous chamber, which constitutes between $3 / 5$ and $2 / 3$ of the total volume of the eye globe, is occupied by the vitreous body. The vitreous is a clear, gel-like material whose viscoelastic behaviour has been analysed in detail by Lee et al. [4]. The vitreous has the important mechanical function of securing retina adherence to the outer layers of the eye. With advancing age, the vitreous often undergoes a liquefaction process; in such a case the formation of liquid cavities within the still gel-like vitreous is often observed. Vitreous liquefaction may even

*Tel.: +390862 434545, Fax: +390862 434548, e-mail: rodolfo@ing.univaq.it 


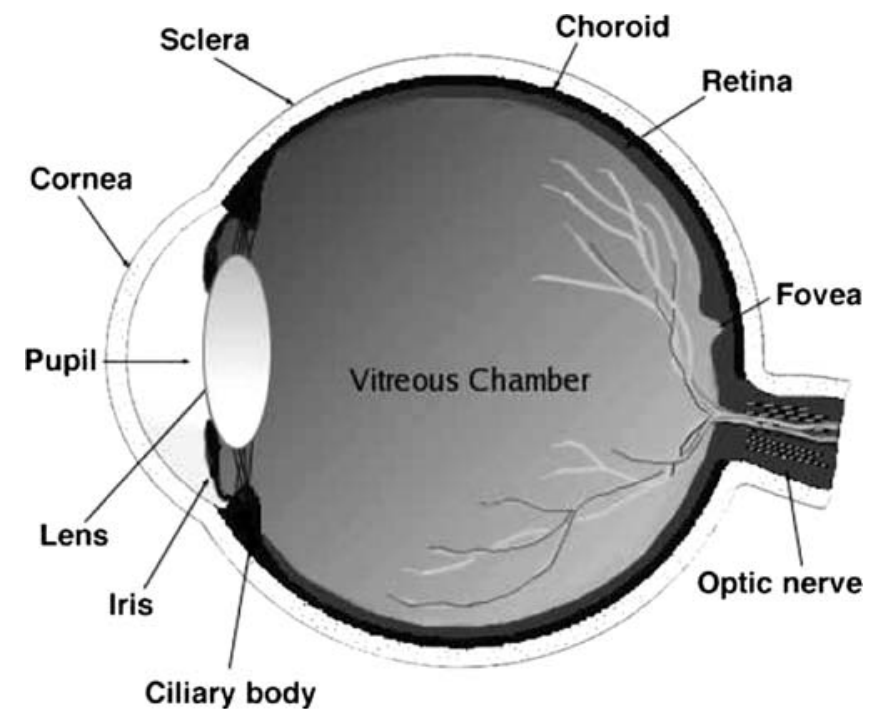

Figure 1. Sketch of the vertical cross section of a human eye.

involve the whole vitreous chamber and it is by far more frequent in myopic eyes. It is worth noting that, after surgery (vitrectomy), the vitreous body is often replaced by other liquids, typically silicon oils, which are kept for a limited time within the chamber (at most some months) to be then removed and naturally replaced by aqueous humour.

Most of the eye globe volume is occupied by fluids, therefore many of the problems related with the eye's functioning are strictly of a fluid dynamic nature. Vitreous dynamics has not been extensively studied yet, even though it is generally accepted that vitreous motion within the vitreous chamber plays an important role in the pathogenesis of retinal detachment. It is indeed well known that, once a retinal tear has occurred, the progressive infiltration of liquefied vitreous through the hole, between the retina and the pigment epithelium, may lead to retinal detachment. This mechanism is called 'rhegmatogeneous retinal detachment'.

The most frequent retinal break mechanism is related to the generation of tensile stresses on the retina, however a full mechanical understanding of such a process is far from available. Repetto et al. [7] have recently proposed an analytical model attempting to interpret one of the possible mechanisms whereby tensile stresses may be generated on the retina, possibly leading to a retinal break. The above authors have studied the dynamics of liquefied vitreous in the presence of vitreous membranes. Their results suggest that vitreous membrane oscillations may be resonantly excited by small-amplitude, high-frequency eye movements, called 'microsaccades', which may thus induce high stresses on the retina and eventually cause its detachment.

Lindner [5] made the first attempt to study the mechanics of retinal detachment, once a retinal tear has occurred, and investigate its link to vitreous dynamics. The author performed a series of experiments with the purpose of studying the fluid dynamics of the vitreous body, induced by eye movements. The experimental apparatus consisted of a cylindrical glass container, filled with water, whose inner wall was covered by a thin transparent membrane. In the membrane a small tear was produced 
and the mechanism of the detachment of the membrane from the container wall was described. It turned out that translational eye movements do not have any effect on membrane detachment which is on the other hand strongly influenced by eye rotations.

The seminal work by Lindner [5] has been reconsidered by Rosengren and Östrelin [8] who conducted a series of experiments on vitreous dynamics, again employing a cylindrical container. The authors stress the importance of obtaining a detailed description of vitreous flow field within the vitreous chamber in order to understand the physical mechanism of retinal detachment. They also underline the importance of the lens, whose presence confers an irregular shape to the chamber and hence may significantly modify flow field characteristics.

While the above contributions point out some basic features of the process, from the fluid mechanics point of view, however, they have serious limitations. In particular the scale effects arising from the magnified dimensions of the chamber model adopted and from the viscosity of the fluid used are not accounted for. Moreover, the authors consider a cylindrical domain whereas, clearly, the simplest geometry which best approximates the shape of the vitreous chamber is the sphere.

Recently, David et al. [3] have reconsidered the problem of vitreous dynamics induced by eye movements, overcoming some of the above limitations. The authors present an analytical model of vitreous motion induced by periodic rotations of the eye globe, modelling the vitreous as a viscoelastic material of Maxwell/Voigt type, with the mechanical properties reported by Lee et al. [4]. The geometry of the domain is spherical and the model is based on the a-priori restrictive assumption of purely azimuthal flow field; in other words the velocity vectors are taken to lie anywhere on planes perpendicular to the axis of rotation. The latter hypothesis implies that the secondary flow possibly induced by the presence on nonlinear terms in the equation of motion is negligible. Numerical simulations performed by the above authors for the case of a Newtonian fluid support the validity of the latter assumption, at least in the range of the controlling parameters typical for the normal motion of the human vitreous. Results obtained by David et al. [3] suggest that the elastic component of the vitreous behaviour plays a fairly minor role. Results also show that the tangential stress induced on the retina increases linearly with the vitreous chamber radius, a finding which is interpreted as a possible explanation of why myopic eyes, which are characterised by a larger size, are much more frequently subject to retinal detachment.

The purely azimuthal nature of the flow field arises also from the quite severe constraint whereby, in the model proposed by David et al. [3], the vitreous chamber is taken to be perfectly spherical. In fact the motion of a rotating sphere may drive fluid motion only through the viscous stresses acting at the wall. However, the shape of the anterior part of the vitreous chamber of a real eye differs significantly from a sphere due to the presence of the lens as shown in Figure 2. The deviation of the chamber shape from that of a sphere may play an important role on the flow field induced in the vitreous, in particular when the vitreous is liquefied and, thus, characterised by a relatively low viscosity. Under these circumstances a perfect sphere subject to a rapid rotation of short duration drives a fluid motion that remains confined within a thin boundary layer adjacent to the wall. In the real geometry, motion is also driven by the non uniform pressure field generated in the interior of the globe by wall rotations 
due to the non-sphericity of the domain. The latter mechanism also leads to the existence of regions where the flow field at the side wall is decelerated, a feature which can induce boundary layer separation.

In the present contribution we develop the above ideas and investigate analytically the vitreous motion induced by eye rotations, considering the real shape of the vitreous chamber and assuming the vitreous to be liquefied. We take advantage of the fairly low viscosity of liquefied vitreous to assume that the boundary layer is thin and the core flow is irrotational. The vitreous chamber is modelled as a weakly deformed sphere. The model provides a detailed description of the flow and pressure fields within the eye globe under the above conditions. In spite of its simplicity the present approach allows to point out the generation of a fully three dimensional flow field, has the advantage of allowing a completely analytical treatment of the problem and will serve as the suitable starting point for more refined investigations.

Before describing the present model it is worth recalling some basic features of rapid eye rotations, called 'saccadic eye movements' (the interested reader is referred to Becker [1], for a detailed overview of the subject). Saccades are eye movements that rapidly redirect the eyes from one point to another. The main characteristics of a saccadic eye movement are: (i) an extremely intense angular acceleration (up to $30000 \mathrm{deg} / \mathrm{s}^{2}$ ) inducing a rapid movement of the eye, (ii) a comparatively less intense deceleration which is nevertheless able to induce a very fast arrest of the rotation and (iii) an angular peak velocity increasing with the saccade amplitude up to a saturation value ranging about $400-600 \mathrm{deg} / \mathrm{s}$. The maximum amplitude of a saccade is about $50^{\circ}$ though most eye rotations have amplitudes smaller than $20^{\circ}$. Becker [1] reports relationships between saccade duration and amplitude; a saccade duration is at most of the order of a tenth of a second.

The paper is organised as follows. In the next section the mathematical problem is formulated, Section 3 describes the solution procedure while results of the model are presented and discussed in Section 4. Finally, some concluding remarks are reported in Section 5.

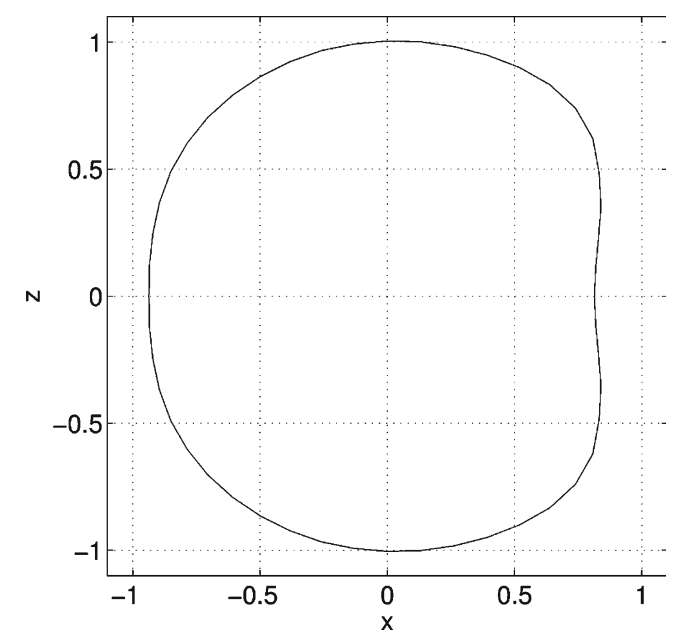

Figure 2. Vertical cross section of the considered domain. 


\section{Formulation of the Problem}

The fluid motion induced by saccadic eye movements within the vitreous chamber is studied. The vitreous is modelled as a Newtonian incompressible fluid characterised by low viscosity. As discussed in the Introduction, the latter hypothesis applies to the case of liquefied vitreous or to the case that the vitreous chamber is filled with aqueous humour after surgery. The eye globe is modelled as a rigid deformed sphere rotating about its vertical axis. The shape of the domain has been obtained from images of the human vitreous chamber. Obviously, the vitreous chamber shape is not uniquely defined, nevertheless there are recurring features which are properly accounted for in the present model, namely both the receding anterior part of the eye, corresponding to the lens, and the fact that, under physiological conditions, the chamber is flattened in the anterior-posterior direction. In Figure 2 the vertical cross section of the domain considered herein is shown. The three dimensional body modelling the vitreous chamber is obtained through a rotation of the above cross-section about its axis of symmetry and is reproduced in Figure 3.

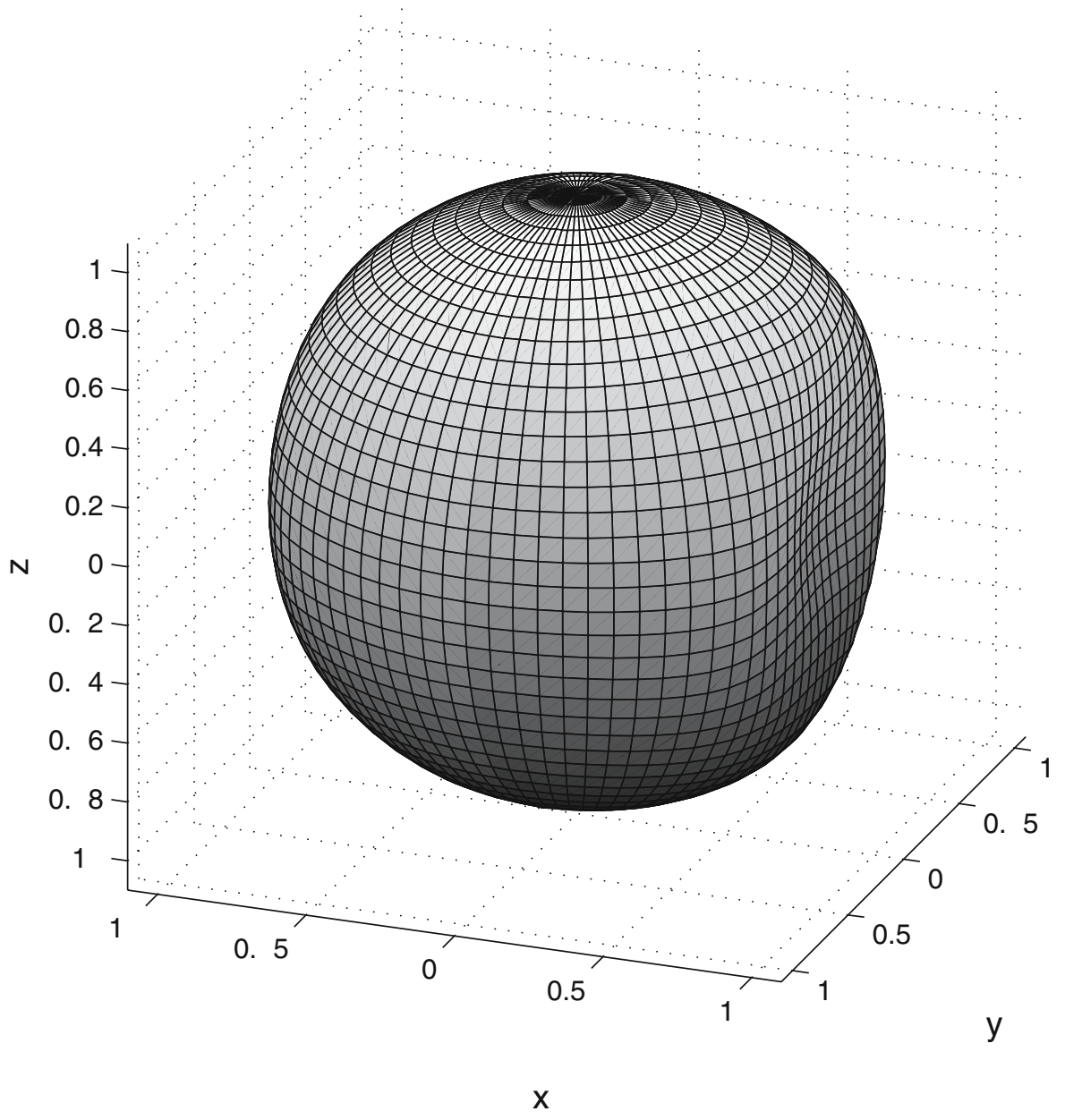

Figure 3. Three dimensional view of the considered domain. 


\section{Rodolfo Repetto}

If we consider a saccadic movement starting from rest, for small times the viscous layer at the wall spreads over a thickness of the order of $\sqrt{v t^{*}}$, where $v$ and $t^{*}$ denote the kinematic viscosity of the fluid and time, respectively ${ }^{1}$. As pointed out in the Introduction, saccades are fairly rapid movements, for instance, according to the relationships proposed by Becker [1], a large saccade with an amplitude of $40^{\circ}$ has a duration of $0.12 \mathrm{~s}$. In the case of a low viscosity fluid, say comparable with water (the aqueous humour has a kinematic viscosity of $0.75 \cdot 10^{-6} \mathrm{~m}^{2} / \mathrm{s}$, Beswick and McCulloch [2]), and on a time scale typical of a saccadic movement the boundary layer thickness is then expected to be of the order of $3 \div 4 \cdot 10^{-4} \mathrm{~m}$, i.e., it keeps much smaller than the eye radius that, in physiological conditions, is approximatively equal to $0.012 \mathrm{~m}$. Herein the attention is focused to the lowest approximation, the viscous boundary layer ignored and the flow assumed to be irrotational. As a matter of fact, the validity of the latter hypothesis implicitly assumes that the boundary layer does not separate during a saccadic movement. Later in the paper an attempt is made to estimate the possible occurrence of boundary layer separation in the case of two dimensional flow and impulsive rotation of the container. In case of larger values of the fluid viscosity viscous effects become more important, as discussed by David et al. [3] for the case of a periodically rotating spherical domain. However, for small saccadic movements of short duration, even in the case of fluid viscosity comparable to that of the physiological vitreous $\left(v \sim 10^{-4}\right.$, from Lee et al. [4]), viscous effects are felt at a distance of a few millimetres from the chamber wall and the flow in the most interior part of the domain is still expected to be irrotational, and thus essentially governed by the chamber's shape. In this respect the solution derived with the present approach helps to isolate and understand the role of the irregularity of chamber shape on the fluid motion. In order to incorporate both the effects of viscosity and domain geometry a fully numerical solution of the Navier-Stokes equations would be required.

The velocity potential $\Phi^{*}$ is defined as

$$
\mathbf{u}^{*}=\nabla \Phi^{*}
$$

where $\mathbf{u}^{*}$ denotes velocity. Fluid incompressibility implies that the velocity potential must be a harmonic function, i.e.

$$
\nabla^{2} \Phi^{*}=0 \text {. }
$$

The mathematical problem is formulated referring to a fixed frame and employing a system of polar spherical coordinates $\left(r^{*}, \vartheta, \phi\right)$ (Figure 4$)$. The boundary conditions impose vanishing flux through the eye wall, defined by a radial coordinate $R^{*}\left(\vartheta, \phi^{*}, t^{*}\right)$. The equations governing the fluid flow can then be written in the form

$$
\begin{aligned}
& \frac{\partial}{\partial r^{*}}\left(r^{* 2} \frac{\partial \Phi}{\partial r^{*}}\right)+\frac{1}{\sin \vartheta} \frac{\partial}{\partial \vartheta}\left(\sin \vartheta \frac{\partial \Phi^{*}}{\partial \vartheta}\right)+\frac{1}{\sin ^{2} \vartheta} \frac{\partial^{2} \Phi^{*}}{\partial \phi^{2}}=0, \\
& -\frac{\partial R^{*}}{\partial t^{*}}+\frac{\partial \Phi^{*}}{\partial r^{*}}-\frac{1}{r^{* 2}} \frac{\partial \Phi^{*}}{\partial \vartheta} \frac{\partial R^{*}}{\partial \vartheta}-\frac{1}{r^{* 2} \sin ^{2} \vartheta} \frac{\partial \Phi^{*}}{\partial \phi} \frac{\partial R^{*}}{\partial \phi}=0 \quad\left[r^{*}=R^{*}\left(\vartheta, \phi-\alpha\left(t^{*}\right)\right)\right],
\end{aligned}
$$

${ }^{1}$ Throughout the paper superscript asterisks denote dimensional variables that will later be made dimensionless. 


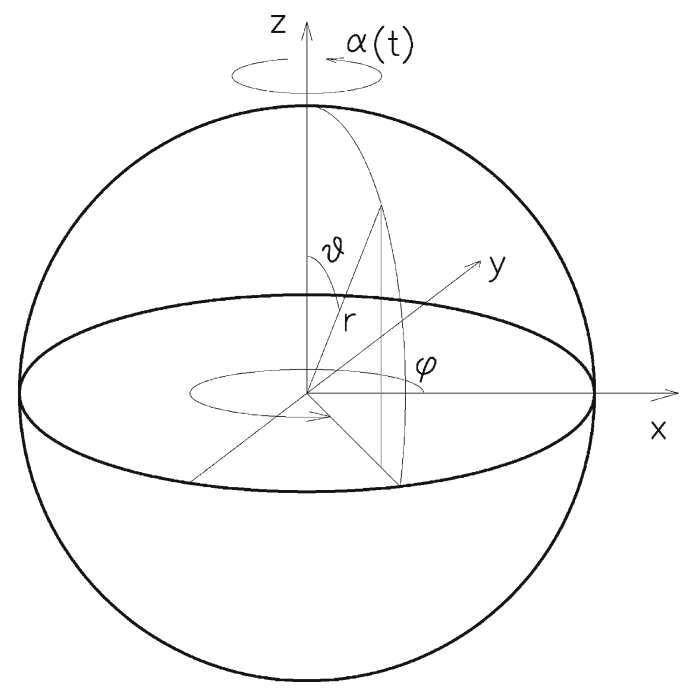

Figure 4. System of coordinates adopted.

where $\alpha\left(t^{*}\right)$ denotes the angle of rotation of the globe with respect to a reference position. Finally, the dynamic pressure $p^{*}$ enters the problem through the Bernoulli equation which, for an unsteady irrotational flow, holds throughout the whole domain and reads

$$
\frac{\partial \Phi^{*}}{\partial t^{*}}+\frac{p^{*}}{\rho}+\frac{1}{2}\left[\left(\frac{\partial \Phi^{*}}{\partial r^{*}}\right)^{2}+\left(\frac{1}{r^{*}} \frac{\partial \Phi^{*}}{\partial \vartheta}\right)^{2}+\left(\frac{1}{r^{*} \sin \vartheta} \frac{\partial \Phi^{*}}{\partial \phi}\right)^{2}\right]=f\left(t^{*}\right)
$$

with $\rho$ fluid density and $f\left(t^{*}\right)$ an arbitrary function which can be set to vanish by suitably redefining the potential function.

The problem is made dimensionless as follows

$$
\begin{aligned}
& (r, R)=\frac{\left(r^{*}, R^{*}\right)}{\mathcal{R}}, \\
& \Phi=\frac{\Phi^{*}}{\Omega_{p} \mathcal{R}^{2}}, \\
& p=\frac{p^{*}}{\rho \Omega_{p}^{2} \mathcal{R}^{2}}, \\
& t=\Omega_{p} t^{*}
\end{aligned}
$$

where $\mathcal{R}$ is the radius of a sphere to be precisely defined, and $\Omega_{p}$ is the peak angular velocity of the saccadic movement.

At this stage it is convenient to perform the following change of coordinates

$$
\varphi=\phi-\alpha(t)
$$

whereby the position of the eye wall is no longer time-dependent. 

form

Using (6)-(10) equations (3)-(5) can be written in the following dimensionless

$$
\begin{aligned}
& \frac{\partial}{\partial r}\left(r^{2} \frac{\partial \Phi}{\partial r}\right)+\frac{1}{\sin \vartheta} \frac{\partial}{\partial \vartheta}\left(\sin \vartheta \frac{\partial \Phi}{\partial \vartheta}\right)+\frac{1}{\sin ^{2} \vartheta} \frac{\partial^{2} \Phi}{\partial \varphi^{2}}=0, \\
& \dot{\alpha} \frac{\partial R}{\partial \varphi}+\frac{\partial \Phi}{\partial r}-\frac{1}{r^{2}} \frac{\partial \Phi}{\partial \vartheta} \frac{\partial R}{\partial \vartheta}-\frac{1}{r^{2} \sin ^{2} \vartheta} \frac{\partial \Phi}{\partial \varphi} \frac{\partial R}{\partial \varphi}=0 \quad[r=R(\vartheta, \varphi)], \\
& \frac{\partial \Phi}{\partial t}-\dot{\alpha} \frac{\partial \Phi}{\partial \varphi}+p+\frac{1}{2}\left[\left(\frac{\partial \Phi}{\partial r}\right)^{2}+\left(\frac{1}{r} \frac{\partial \Phi}{\partial \vartheta}\right)^{2}+\left(\frac{1}{r \sin \vartheta} \frac{\partial \Phi}{\partial \varphi}\right)^{2}\right]=0,
\end{aligned}
$$

where a superscript dot denotes derivatives with respect to the dimensionless time.

\section{Solution}

The eye globe can be thought of as a slightly deformed sphere (see Figure 3 ). The function $R(\vartheta, \varphi)$, which defines the shape of the domain is then expressed in the form

$$
R(\vartheta, \varphi)=1+\delta R_{1}(\vartheta, \varphi)
$$

where $\delta R_{1}$ measures the displacement of the actual boundary from the sphere, $R_{1}$ has zero average and $\delta$ represents the absolute value of the maximum displacement. The function $\delta R_{1}$ adopted herein, is plotted in Figure 5 as a function of $\varphi$ for $\vartheta=\pi / 2$. The centre of the sphere has been chosen as the point about which the eye globe rotates. Notice that eye movements do not only involve rotations about a fixed centre: small translations also occur which drive a displacement of the centre of rotation. However, such a displacement is very small and it can be neglected for the present purposes. The function $R_{1}(\vartheta, \varphi)$ can then be expanded in terms of spherical harmonics as follows

$$
R_{1}=\sum_{m=0}^{\infty} \sum_{n=m}^{\infty} a_{m n} \cos (m \varphi) P_{n}^{m}(\cos \vartheta)
$$

where $P_{n}^{m}$ are the associated Legendre functions, defined in terms of the Legendre polynomials $P_{n}$ as follows

$$
P_{n}^{m}(x)=\left(1-x^{2}\right)^{m / 2} \frac{\mathrm{d}^{m}}{\mathrm{~d} x^{m}} P_{n}(x) .
$$

Notice that, as the domain is symmetrical with respect to the plane $y=0$, only the symmetrical Fourier modes $(\cos m \varphi)$ have been included in the expansion (15). Taking advantage of the orthogonality properties of the associated Legendre functions, the coefficients $a_{m n}$ appearing in (15) can be easily computed (see for instance Morse and Feshbach [6], p. 1265) as 


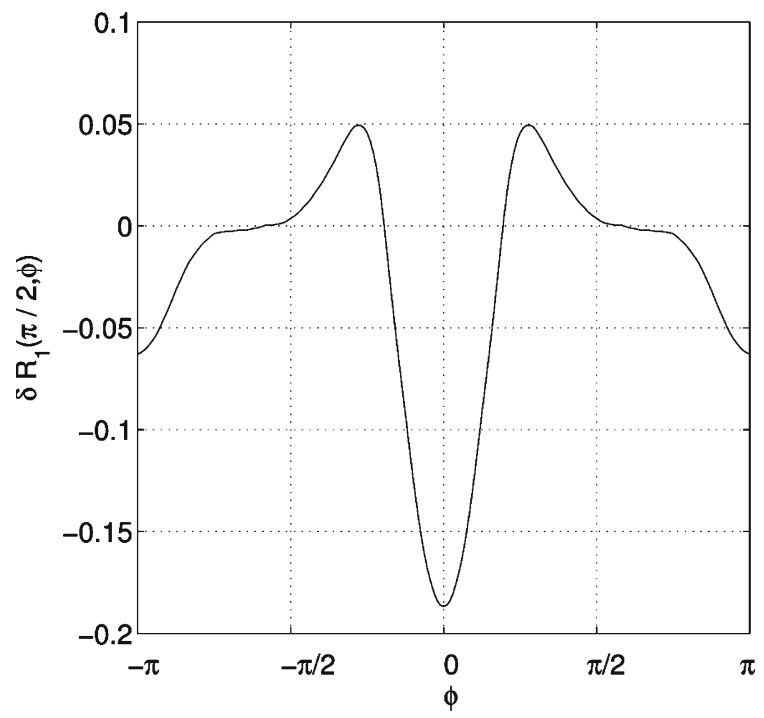

Figure 5. Function $\delta R_{1}(\vartheta, \varphi)$ for $\vartheta=\pi / 2$.

$$
\begin{aligned}
a_{m n}= & \frac{k_{m}(2 n+1)(n-m) !}{4 \pi(n+m) !} . \\
& \cdot \int_{0}^{2 \pi} \int_{0}^{\pi} R_{1}(\vartheta, \varphi) \cos (m \varphi) P_{n}^{m}(\cos \vartheta) \sin \vartheta d \vartheta d \varphi, \\
& k_{0}=1, \quad k_{m}=2, \quad m>0 .
\end{aligned}
$$

In order to reproduce the function $R_{1}$ with a precision of $1 \%, 400$ terms have been employed in the expansion (15).

In the case of the real eye, under physiological conditions, the parameter $\delta$ attains values ranging from 0.15 to 0.2 (see Figure 5). This suggests to set up an expansion of the unknown functions $\Phi$ and $p$ in powers of $\delta$ as follows

$$
\begin{aligned}
& \Phi=\Phi_{0}+\delta \Phi_{1}+\mathcal{O}\left(\delta^{2}\right), \\
& p=p_{0}+\delta p_{1}+\mathcal{O}\left(\delta^{2}\right) .
\end{aligned}
$$

In the following only terms up to the order $\delta$ will be retained in the above expansions. Even though this approach is strictly valid only for vanishingly small values of $\delta$, however the solution is likely to catch most of the features of the real flow field and the linearity allows one to derive the solution in analytical form. The inclusion of further terms in the perturbation solution is obviously possible but it would require heavy algebraic calculations that are not seen to be worth pursuing: a more accurate solution can more easily be obtained through a fully numerical approach.

By substituting from the expansions (19) and (20) into the governing equations (11)-(13), at the first order of approximation $\mathcal{O}\left(\delta^{0}\right)$ we find the trivial solution $\Phi_{0}=0$, $p_{0}=$ constant. In other words, no motion is generated in a viscous fluid filling a rotating sphere if the no slip condition at the wall is not imposed. 


\section{Rodolfo Repetto}

At order $\delta$ the governing equations (11)-(13) reduce to the following form

$$
\begin{aligned}
& \nabla^{2} \Phi_{1}=0, \\
& \frac{\partial \Phi_{1}}{\partial r}=-\dot{\alpha} \frac{\partial R_{1}}{\partial \varphi} \quad(r=1), \\
& p_{1}=-\frac{\partial \Phi_{1}}{\partial t}+\dot{\alpha} \frac{\partial \Phi_{1}}{\partial \varphi} .
\end{aligned}
$$

Equation (15) and the boundary condition (22) suggest to expand the function $\Phi_{1}$ as follows

$$
\Phi_{1}=\sum_{m=0}^{\infty} \sum_{n=m}^{\infty} \Phi_{m n}(r) \sin (m \varphi) P_{n}^{m}(\cos \vartheta) .
$$

Substituting from the latter expansion into the equations (21) and (22), the following ordinary differential equation is obtained

$$
\begin{aligned}
& \frac{\mathrm{d}}{\mathrm{d} r}\left(r^{2} \frac{\mathrm{d} \Phi_{m n}}{\mathrm{~d} r}\right)-n(n+1) \Phi_{m n}=0, \\
& \frac{\mathrm{d} \Phi_{m n}}{\mathrm{~d} r}=a_{m n} \dot{\alpha} m, \quad(r=1), \\
& \Phi_{m n}=0 \quad(r=0) .
\end{aligned}
$$

Notice that the second order equation (25) is to be solved subject to a condition imposing the regularity of the solution at the origin. The condition (27) insures that such a constraint be satisfied. Since the velocity potential is defined up to a constant, any finite value of $\Phi_{m n}$ at the origin would have been chosen. Equation (25) with the boundary conditions (26) and (27) admits of the solution

$$
\Phi_{m n}=a_{m n} \dot{\alpha} \frac{m}{n} r^{n},
$$

hence, the order $\delta$ velocity components read

$$
\begin{aligned}
& u_{r 1}=\sum_{m=0}^{\infty} \sum_{n=m}^{\infty} a_{m n} \dot{\alpha} m r^{n-1} \sin (m \varphi) P_{n}^{m}(\cos \vartheta), \\
& u_{\vartheta 1}=\sum_{m=0}^{\infty} \sum_{n=m}^{\infty} a_{m n} \dot{\alpha} \frac{m}{n} r^{n-1} \sin (m \varphi) \frac{\mathrm{d}}{\mathrm{d} \vartheta} P_{n}^{m}(\cos \vartheta), \\
& u_{\varphi 1}=\sum_{m=0}^{\infty} \sum_{n=m}^{\infty} \frac{a_{m n} \dot{\alpha} \frac{m^{2}}{n} r^{n-1} \cos (m \varphi) P_{n}^{m}(\cos \vartheta)}{\sin \vartheta},
\end{aligned}
$$

where

$$
\begin{aligned}
& \frac{\mathrm{d}}{\mathrm{d} \vartheta} P_{n}^{m}(\cos \vartheta) \\
& \quad=-\sin \vartheta \frac{(n-m+1) P_{n+1}^{m}(\cos \vartheta)-(n+1) \cos \vartheta P_{n}^{m}(\cos \vartheta)}{\cos ^{2} \vartheta-1} .
\end{aligned}
$$


Finally, from the linearised Bernoulli equation (23) the pressure is found to read

$$
p_{1}=\sum_{m=0}^{\infty} \sum_{n=m}^{\infty}-a_{m n} \ddot{\alpha} \frac{m}{n} r^{n} \sin (m \varphi) P_{n}^{m}(\cos \vartheta)+a_{m n} \dot{\alpha}^{2} \frac{m^{2}}{n} r^{n} \cos (m \varphi) P_{n}^{m}(\cos \vartheta) .
$$

It is not surprising that the expression for the pressure involves a term proportional to the angular acceleration of the eye globe and a second term proportional to its angular velocity.

\section{Results and Discussion}

In Figure 6a the absolute flow field induced on the horizontal plane $z=0$ by a counterclockwise rotation of the eye globe is shown. It appears that the geometry of the domain generates a particularly intense flow close to the anterior part of the vitreous chamber (positive values of the $x$ coordinate), where the displacement of the wall profile with respect to the spherical shape is most pronounced. In particular, close to the lens, the velocity is in the opposite direction with respect to the motion of the wall. This is not surprising: indeed, the receding part of the wall at the lens moves upward (see Figure 2a), hence the fluid close to the lens moves in the opposite direction, being forced to abandon the region that the lens is occupying. Figure $6 \mathrm{a}$ shows that a velocity field is also generated in the posterior region of the vitreous chamber, though it is less intense than that occurring close to the lens, while the velocity vectors are again directed in the opposite sense with respect to the wall velocity. Notice that the Reynolds number of the flow (computed with the maximum absolute velocity, the eye radius and the kinematic viscosity of water) assumes values of at most some hundreds; it is therefore reasonable to expect the flow to be laminar. The velocity distribution at the wall suggests that, if the non-spherical character of the domain is accounted for, the shear stress at the wall may be significantly modified with respect to the perfectly spherical case. In order to determine the shear stress at the wall it would be necessary to solve the equation of motion within the boundary layer at the wall, an endeavour which is beyond of the scope of the present work. However, note that the present model provides the matching condition 'at infinity' for the flow in the boundary layer.

In Figure 6b the same flow field is plotted in terms of relative velocity.

In Figure 7 the absolute velocity component $u_{y 1}$ in the vertical plane of equation $y=0$ is shown. Again it clearly appears that the most intense velocities are generated close to the lens and in the posterior part of the eye globe. It is interesting to notice that the isotachies have a different curvature from those relative to the viscous solution for the motion of a Newtonian fluid filling a rotating sphere. This is shown in Figures 8a,b where the absolute velocity component $u_{y}$ obtained through a simplified viscous solution within a sphere is plotted. The sphere is assumed to rotate with a time law reproducing a real saccadic movement and the plots reported in Figures $8 \mathrm{a}, \mathrm{b}$ refer to the time at which the maximum angular velocity is reached by the sphere. The solution has been obtained numerically, adopting the same assumptions employed by David et al. [3], i.e. assuming that the velocity vectors lie everywhere on planes orthogonal to the $z$-axis. The two plots refer to two different values of the fluid viscosity. 


\section{Rodolfo Repetto}
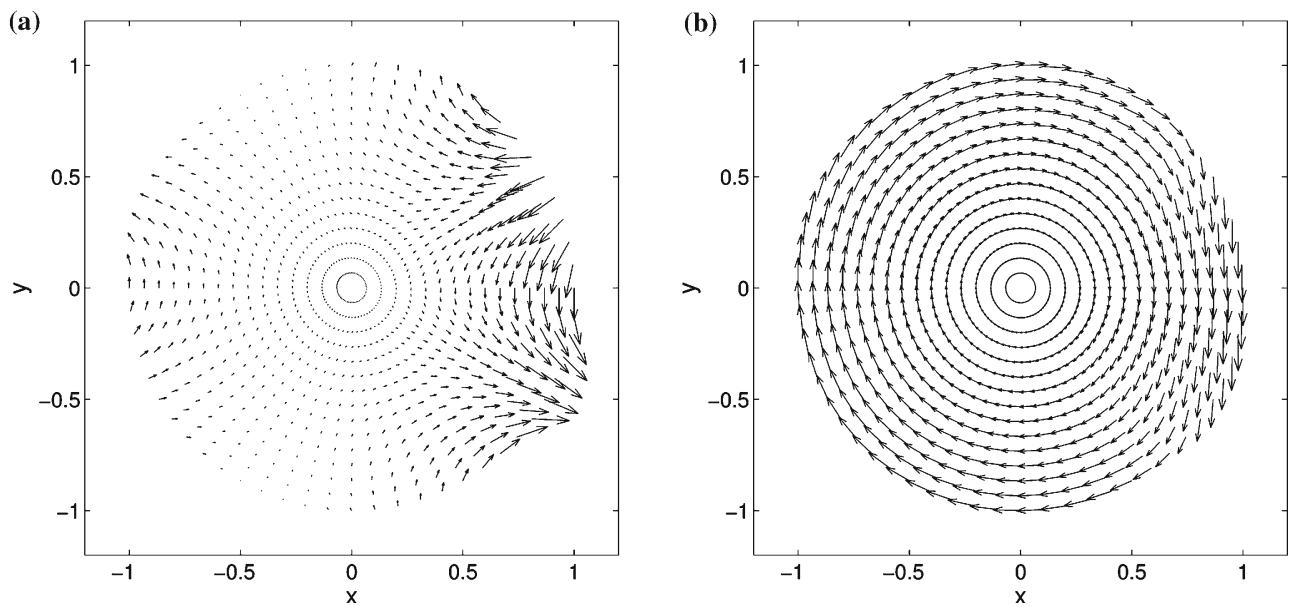

Figure 6. Absolute $\left(\mathbf{u}_{1}\right)$ (a) and relative (b) velocity fields on the horizontal plane $z=0$, induced by a counterclockwise rotation of the eye globe. The relative velocity field has been computed adopting $\delta=0.186$. For graphical reasons the vector lengths in the two plots have different scales. Maximum vector lengths: 2.46 (a), 1.40 (b).

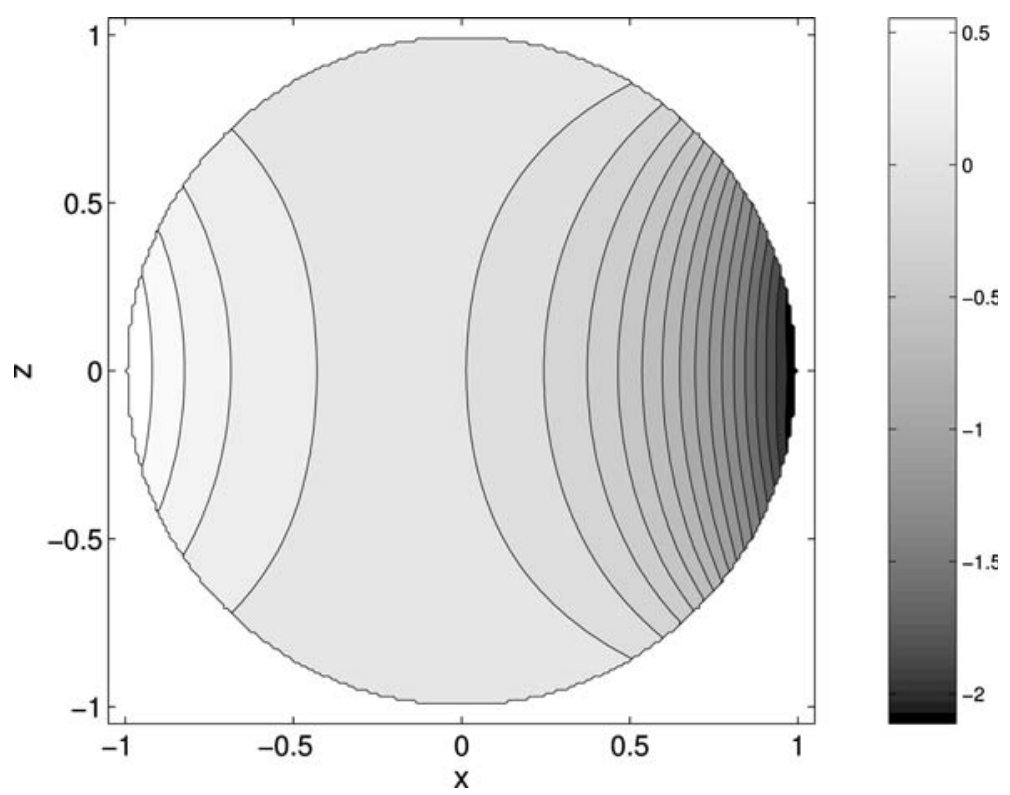

Figure 7. Absolute velocity component $u_{y 1}$ in the plane $y=0$ induced by a counterclockwise rotation of the eye globe.

The flow field generated by the rotation of the eye globe is strongly three-dimensional as shown in Figure 9, where the projection of the absolute velocity field on the vertical plane forming an angle of $30^{\circ}$ with the $x$ axis $(\varphi=\pi / 6,7 / 6 \pi)$ is plotted. The geometry of the domain is responsible for the presence of significant vertical velocity components which would not be generated if the eye globe were purely spherical. In particular, close to the lens, the vertical velocity components are found to be positive in the upper hemisphere and negative in the lower one. Let us point 

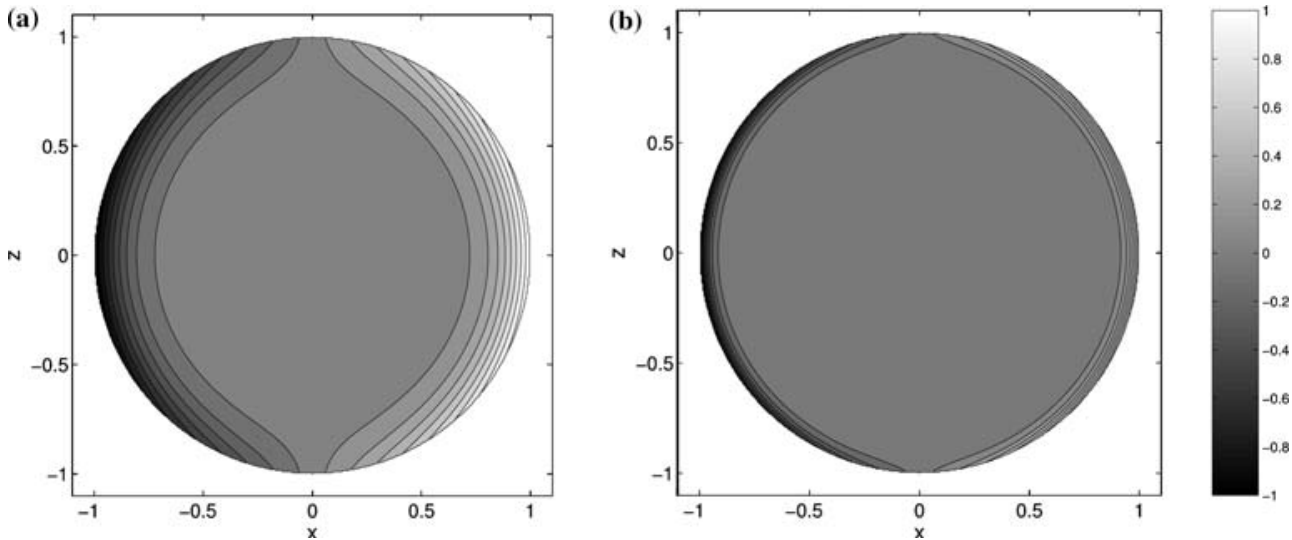

Figure 8. Absolute velocity in the plane $y=0$ induced by a counterclockwise rotation of the eye globe as predicted by a simplified viscous solution within a sphere. The saccade amplitude is $10^{\circ}$, the angular velocity of the sphere is the maximum reached during the saccadic movement and is equal to $371 \% \mathrm{~s}$ (from Becker [1]). (a) $v=10^{-4} \mathrm{~m}^{2} / \mathrm{s}$, (b) $v=10^{-5} \mathrm{~m}^{2} / \mathrm{s}$.

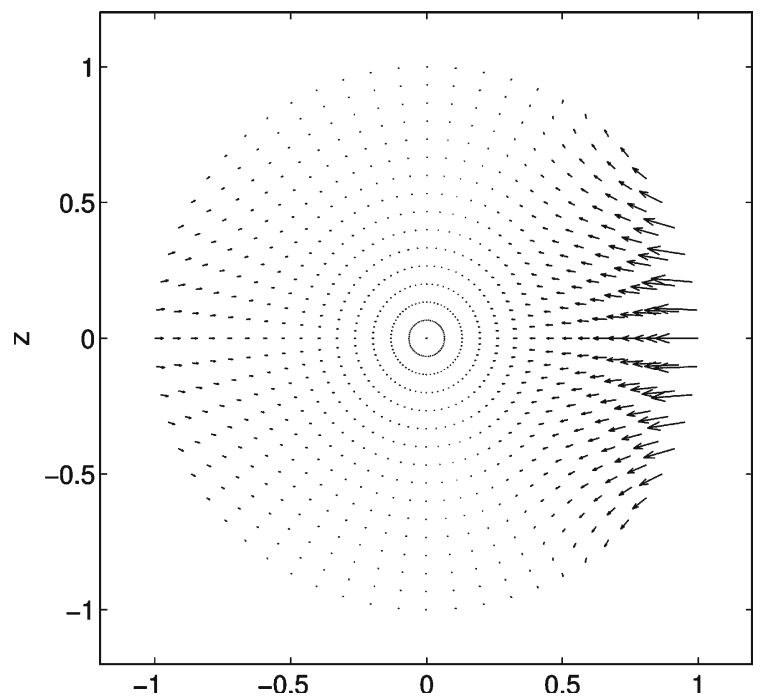

Figure 9. Projection of the absolute velocity field $\mathbf{u}_{1}$ induced by a counterclockwise rotation of the eye globe on the vertical plane $\varphi=\pi / 6, \varphi=7 / 6 \pi$. The vector length scale is the same as that adopted in Figure 6a.

out that the three-dimensional character of the flow may play an important role in producing vitreous mixing.

Finally, in Figures 10a,b, pressure fields are reported on the plane $z=0$. The two plots refer to two different times during a saccadic movement: the initial instant, in which the angular acceleration peaks (a) and the instant at which the angular velocity attains its maximum and the angular acceleration vanishes (b). As suggested by equation (33), in the former case the pressure field on the horizontal plane is anti-symmetric with respect to the $x$ axis while, in the latter case, it is symmetric. The anterior and the posterior regions of the eye globe are characterised by 

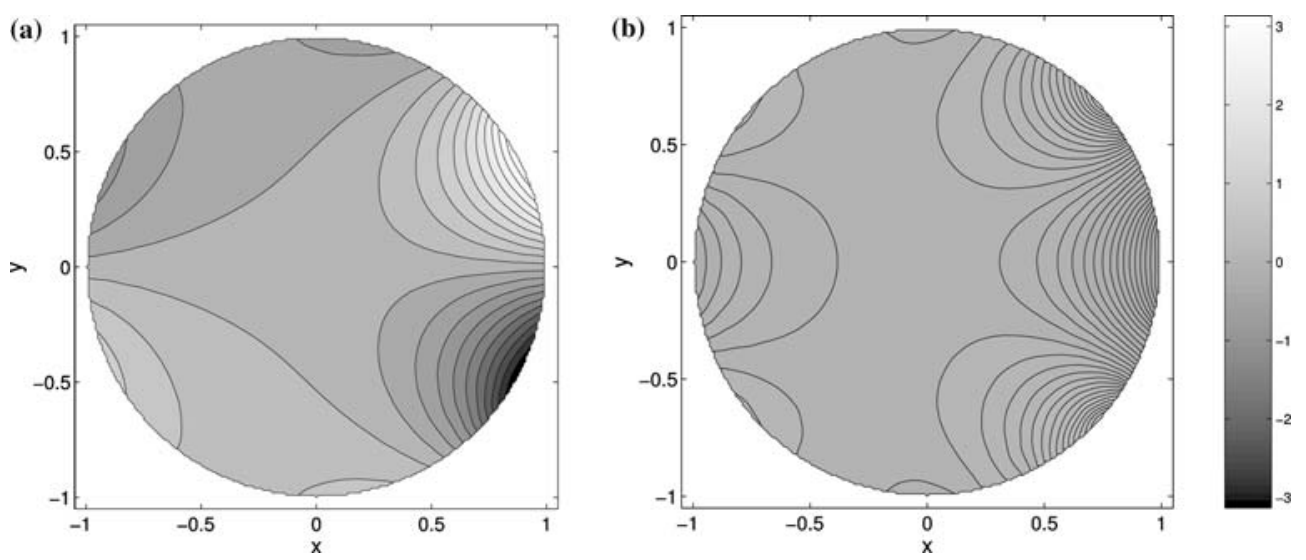

Figure 10. Pressure fields on the plane $z=0$ generated at two different times during a counterclockwise saccadic movement. (a) $\mathrm{d} \alpha / \mathrm{d} t^{*}=0 \rightarrow \dot{\alpha}=0, \mathrm{~d}^{2} \alpha / \mathrm{d} t^{* 2}=30000^{\circ} \mathrm{s}=523.6 \mathrm{rad} / \mathrm{s}^{2} \rightarrow \ddot{\alpha}=4.77$; (b) $\mathrm{d} \alpha / \mathrm{d} t^{*}=\Omega_{p}=600^{\circ} / \mathrm{s}=10.47 \mathrm{rad} / \mathrm{s} \rightarrow \dot{\alpha}=1, \mathrm{~d}^{2} \alpha / \mathrm{d} t^{* 2}=0^{\circ} / \mathrm{s}^{2} \rightarrow \ddot{\alpha}=0$. Dimensional values of angular velocity and acceleration of the eye globe from Becker [1].

the most intense dynamic pressures. Notice however that, during all the saccadic movement, the dynamic pressure assumes everywhere very small values when compared to the static intraocular pressure. Nevertheless, such dynamic pressure varies very rapidly and may be important in the understanding of some aspects of the pathogenesis of retinal detachment. Indeed, it is known that the adhesion between the retina and the pigment epithelium is partly due to a pressure gradient between the vitreous chamber and the eye's outer layers. If the pressure in such layers does not adapt rapidly to pressure variations within the vitreous chamber it is possible that, during a saccadic movement, the pressure gradient changes sign, thus decreasing the adhesive force between the retina and the pigment epithelium. The above speculations, which are based on purely mechanical considerations, will require to be substantiated by clinical evidence and measurements which are not yet available.

The proposed solution is correct only under the assumption that no boundary layer separation occurs during a saccadic movement. A glance at the flow field reported in Figure $6 \mathrm{~b}$ shows that, downstream of the lens, the fluid speed at the wall decreases. A decreasing speed distribution along a rigid wall is known to favour boundary layer detachment. In order to verify whether such a boundary layer separation may actually occur under the conditions examined above, the solution of the three-dimensional unsteady boundary layer equations is required. However, an indication of the possible occurrence of boundary layer separation can be simply obtained considering a two-dimensional flow (on the plane $z=0$ ) driven by an impulsive rotation from rest of the domain. Under such hypotheses one can employ the classical procedure reported by Stuart [9] and obtain an estimate of the minimum angle of rotation for which boundary layer separation occurs, as a function of the maximum negative value of the spatial derivative of the velocity along the wall profile. The method consists of an iteration procedure and its physical basis is the observation that, initially, the boundary layer is extremely thin and the main contribution to the time derivative of velocity in the boundary layer equations is 
due to viscous diffusion. A first approximation solution, for small values of $t$, is obtained solving a purely diffusive equation. The computed values of the velocity are used to evaluate the convective terms for a second order solution. The procedure can be iterated to improve the solution. However, already at the second order of approximation, it is possible to estimate the possible occurrence of back-flow within the boundary layer and the consequent boundary layer separation. In the case of the two-dimensional domain presented in Figure 2, rotations of the eye globe with amplitude larger than about $15^{\circ}$ are expected to produce boundary layer separation. The predicted value only provides a rough, and probably underestimated, prediction of the amplitude of a saccade that may produce boundary layer separation. Nevertheless, the above result suggests that, in the case of low viscosity vitreous, boundary layer separation may indeed occur during large amplitude saccadic movements, with consequent formation of a wake region within the eye globe. This points the need for further developments of the present work to investigate the structure and evolution of the viscous boundary layer driven by the actual rotation of the wall.

\section{Conclusions}

Indications from the medical literature suggest that retinal detachment occurrence and progression is largely related to mechanical phenomena. This underlines the need for mechanical research on such a topic. However, up to now, very few contributions exist and we are far from a true understanding of retinal detachment mechanisms. This is also due to the fact that, from the mechanical point of view, the problem is extremely complicated, such a complexity mainly arising from the rheological properties of the real vitreous, both in physiological and in certain pathological conditions. In particular it is well known that the vitreous may be tightly anchored to the retina in specific areas and, especially in myopic eyes, the existence of such adherence points is the major cause of formation of retinal tears which may lead to rhegmatogeneous retinal detachments.

In order to face such a complex system researchers have been up to now forced to focus their studies on fairly simplified conditions, with the aim of understanding basic phenomena taking place within the vitreous chamber. The present work follows this line of reasoning, the main goal of the paper being to gather understanding on the role of the non-sphericity on the vitreous chamber on the vitreous motion. This problem has not been addressed in earlier contributions. With the aim of obtaining a fully analytical solution, our attention has been restricted to the case of liquefied vitreous, i.e., conditions in which the vitreous body has lost its elastic properties and is characterised by a fairly low viscosity. Such conditions may indeed occur, both in elderly and in cases of vitreoctomized eyes.

The problem of understanding how the liquefied vitreous flows within the eye in response to eye movements is of great interest because of its connection with rhegmatogeneous retinal detachment. This type of detachment is always caused by a progressive infiltration of liquefied vitreous through a retinal tear, though the vitreous is not necessarily completely liquefied. In order to understand the mechanism of 


\section{Rodolfo Repetto}

liquefied vitreous infiltration through a retinal break, which is an important open problem to be addressed by future research, it is obviously necessary to obtain a detailed description of the flow and pressure fields within the vitreous chamber. In this respect the main result of the present work is to evidence the fact that the real shape of the domain plays a significant role, being responsible for an intense flow with remarkably different characteristics from the viscous flow occurring within a rotating sphere. Stresses on the retina induced by such a flow are also expected to differ substantially form the viscous stresses generated in the spherical case. Therefore, future more refined models of the vitreous motion will have to account for the real shape of the vitreous chamber.

An important implication of the results presented in the paper is the significant three-dimensionality of the flow field. David et al. [3] state that the weak secondary flow that takes place within a periodically rotating sphere may be important to induce a complete recirculation of the vitreous over large timescales. The results of the present work show that the non-sphericity of the vitreous chamber is much more efficient in producing vitreous mixing.

Finally, by simple considerations it has been shown that the real shape of the vitreous chamber may induce boundary layer separation and the generation of complex three-dimensional flow and pressure fields. The possible existence of such complex flows, which can be studied only through a direct numerical solution of the equations of motion, has never been considered in previous studies and may have important clinical implications, for instance for the choice of suitable tamponade fluids to be used after vitrectomy.

\section{Acknowledgements}

This work has been developed within the framework of the project FIRB2001 (RBAU01Z44F005) co-funded by the MIUR. The author wishes to thank Dr. Andrea Scupola for providing useful advices on the clinical aspects of the problem. The author is also grateful to Alessandra Mastroianni who contributed to a preliminary version of the present work during her Master Thesis.

\section{References}

1. Becker, W., 'Metrics', in: Wurtz and Goldberg (eds.), The neurobiology of saccadic eye movements. Elsevier Science Publisher BV (Biomedical Division), 1989.

2. Beswick, J.A. and McCulloch, C., 'Effect of hyaluronidase on the viscosity of the aqueous humour', Brit. J. Ophthamol. 40 (1956) 545-548.

3. David, T., Smye, S., Dabbs, T. and James, T., 'A model for the fluid motion of vitreous humour of the human eye during saccadic movement', Phys. Med. Biol. 43 (1998) 1385-1399.

4. Lee, B., Litt, M. and Buchsbaum, G., 'Rheology of the vitreous body. Part I: viscoelasticity of human vitreous'. Biorheology 29 (1992) 521-533.

5. Lindner, K., 'Über die Herstellung von Modellen zu Modellversuchen der Netzhautabhebung', Klin. monatsbl. Augenh 90 (1933) 289-300.

6. Morse, P.M. and Feshbach, H., Methods of Theoretical Physics. McGraw-Hill, New York, 1953.

7. Repetto, R., Ghigo, I. Seminara, G. and Ciurlo, C., 'A simple hydro-elastic model of the dynamics of a vitreous membrane', J. Fluid Mech. 503 (2004) 1-14. 
8. Rosengren, B. and Östrelin, S., 'Hydrodynamic events in vitreous space accompanying eye movements'. Ophtalmologica 173 (1976) 513-524.

9. Stuart, J.T., 'Unsteady Boundary Layers', In: Rosenhead L. (ed.); Laminar Boundary Layers, Clarendon Press, Oxford, 1963. 